pip, a nucleus. Do we infer from the work of Hernquist and Barnes that it will often be kinematically decoupled from its surrounding halo? More than half of all galactic disks develop 'warps' at radii of the order of 10 kiloparsecs and a popular explanation of the phenomenon (A. Toomre, IAU Symp. 100, 177-186; 1983) is precisely that the spin axes of galaxies are misaligned with those of their halos. Moreover, rings of gas are sometimes found around disk galaxies, the spin axes of the ring and the disk being roughly perpendicular just as in Hernquist and Barnes's simulation.

Finally, a potential problem with the cold-dark-matter model is that large halos that have grown by repeated accretion would be expected to have very small core radii, if any. But such coreless halos are probably incompatible with the observed rotation curves of many disk galaxies. When two halos merge, do the visible galaxies act like the black holes in the calculations by Ebisuzaki et al. and increase the core radius of the halo at each stage in the merging process?

James Binney is in the Sub-department of Theoretical Physics, Oxford University, 1 Keble Road, Oxford OX1 3NP, UK.

\title{
NEUROSCIENCE
}

\section{Vehicles of inactivation}

\section{Solomon H. Snyder}

FOUR reports in Nature ${ }^{1}$ and Science ${ }^{2-4}$, with another to come in Proceedings of the National Academy of Sciences ${ }^{5}$, together announce the cloning of two of the major transporter molecules for neurotransmitters. These developments molecular studies of transmitter transport; they will, one hopes, add further impetus to the drive for psychotherapeutic innovation.

After their synaptic release, biogenic amine and amino-acid neurotransmitters are inactivated by transport back into the nerve terminals that had released them. Therapeutic agents (such as tricyclic and related antidepressants) and drugs of abuse (such as cocaine) seem to act by blocking this transport for one or another biogenic amine. Molecular cloning, which has provided deep insight into postsynaptic receptors, has quite recently been applied to presynaptic neurotransmitter transporters or 'uptake receptors'. Now, essentially simultaneously, two groups report the cloning of serotonin transporters ${ }^{1,2}$, and three

As the first neurotransmitter to be are the latest fruit of the explosion in the cloning of dopamine transporters ${ }^{3-5}$.

characterized, acetylcholine historically provided the model for transmitter disposition. Because acetylcholine is inactivated through enzymatic degradation by acetylcholinesterase, it was assumed for many years that synaptic inactivation for all transmitters would involve enzymatic breakdown. In the late 1950s and early 1960s, Julius Axelrod and colleagues overturned this idea, showing that transmitter re-uptake was the main inactivator for catecholamines ${ }^{6}$. Monitoring of radiolabelled transmitter accumulation by isolated nerve terminals (synaptosomes) extended study of the importance of uptake to other biogenic amines and amino-acid transmitters ${ }^{7}$. Re-uptake is now regarded as the universal mechanism of transmitter inactivation, enzymatic degradation applying only to acetylcholine and neuropeptides.

As with many postsynaptic receptors, the molecular cloning of presynaptic transporters resulted from isolation of the relevant transport protein, a task first accomplished by Kanner and associates, who purified the $\gamma$-aminobutyric acid (GABA) transporter ${ }^{8}$ and then cloned its complementary DNA (ref. 9).

\begin{tabular}{llll} 
Neurotransmitter transporters & & \\
\hline GABA & Noradrenaline & Dopamine & Serotonin \\
67,000 & 69,000 & 69,000 & $73,000^{1}$, \\
& & & $68,000^{2}$ \\
599 & 617 & 619 & $653^{1}, 607^{2}$ \\
4.2 & $5.8,3.6$ & 3.7 & $3.1^{1}, 3.7^{2}$ \\
3 & 3 & 4 & 2 \\
1 & 0 & 0 & 1
\end{tabular}

All four transporters display 12 transmembrane domains with both $\mathrm{C}$ and $\mathrm{N}$ termini being cytoplasmic. The absence in noradrenaline and dopamine transporters of canonical sequences for phosphorylation by cyclic-AMP-dependent protein kinase (PKA) does not rule out PKA phosphorylation, as these two transporters possess less stringent consensus sites for PKA phosphorylation. Differences in length of the two serotonin transporter proteins reflect isolated base differences in sequences from the two laboratories ${ }^{1,2}$ which shift the reading frame. Whether cloning errors are involved or whether there are real differences between the serotonin transporters of rat brain ${ }^{1}$ and rat basophilic leukocytes ${ }^{2}$ is not apparent.
GABA transport was expressed by transfecting the single cloned cDNA encoding a protein of relative molecular mass 67,000 , which, like the glucose and other transporters, displays 12 transmembrane regions, but does not show sequence similarity to these other transporters. Amara and colleagues used expression cloning strategies to isolate and sequence cDNA for the noradrenaline transporter ${ }^{10}$. The 70 per cent similarity in amino-acid sequence of the two transporters encouraged several groups to adopt polymerase chain reaction (PCR) technology based on conserved elements of the GABA and noradrenaline transporters, resulting in the cloning of dopamine and serotonin transporters; a comparison of the four is shown in the table. The $60-70$ per cent similarity in the amino-acid sequences of GABA and the three amine transporters portends the cloning in the near future of all known transmitter transporters.

The cloned transporters should help add to our knowledge of drug action; indeed, the papers on the dopamine ${ }^{3-5}$ and noradrenaline ${ }^{10}$ transporters give headline prominence to the transporters' sensitivity to cocaine. Among a series of cocaine derivatives, inhibition of dopamine uptake correlates best with behavioural reinforcing effects in animals ${ }^{11}$. But the potencies of cocaine at noradrenaline, dopamine and serotonin transporters are quite similar, so that at doses ingested by humans all three uptake systems will be influenced. Among therapeutic agents, antidepressants are the most prominent drugs that act by blocking transmitter uptake, with resultant potentiation of noradrenaline and serotonin as a therapeutic mechanism. Inhibition of GABA and glycine uptake should provide sedative, antianxiety and anticonvulsant effects, but adequately potent and safe agents have not yet appeared. With hard work and ingenuity, and maybe a little luck, it may not be long before they do.

Solomon H. Snyder is in the Departments of Neuroscience, Pharmacology and Molecular Sciences, and Psychiatry, The Johns Hopkins University School of Medicine, $725 \mathrm{~N}$. Wolfe Street, Baltimore, Maryland 21205, USA.

1. Blakely, R. D. et al. Nature 354, 66-70 (1991)

2. Hoffman, B. J., Mezey, E. \& Brownstein, M. J. Science 254, 579-580 (1991)

3. Shimada, S. et al. Science 254, 576-578 (1991).

4. Kilty, J. E., Lorang, D. \& Amara, S. G. Science 254, 578-579 (1991)

5. Usdin, T., Mezey, E., Chen, C., Brownstein, M. J. \& Hoffman, B. J. Proc. natn. Acad. Sci. U.S.A. (in the press)

6. Axeirod, J. Science 173, 598-606 (1971)

7. Snyder, S. H. Biol. Psychiat. 2, 367-389 (1970).

8. Radian, R., Bendahan, A. \& Kanner, B. I. J. biol. Chem 261, 15437-15441 (1986)

9. Guastella, J. et al. Science 249, 1303-1306 (1990)

10. Pacholczyk, T., Blakely, R. D. \& Amara, S. G. Nature 350, 350-354 (1991)

11. Ritz, M. C., Lamb, R. J., Goldberg, S. R. \& Kuhar, M. J. Science 237, 1219-1223 (1987) 